\title{
AGRESSIVIDADE FISCAL E INVESTIMENTOS NO MERCADO ACIONARIO BRASILEIRO
}

Rodolfo Fabriz Marchesi'

Eduardo José Zanoteli

Resumo:

Objetivo: Partindo da premissa de que os benefícios do planejamento tributário são gerados sob a forma de reservas financeiras provenientes da redução dos gastos tributários, o objetivo do presente artigo foi analisar a relação das práticas de agressividade fiscal com o investimento das empresas.

Método: A amostra é composta por 2.752 observações, distribuídas em empresas de capital aberto de 13 setores econômicos. Os dados coletados referem-se aos anos de 2010 a 2018. Por meio da técnica de Regressão Quantílica buscou-se avaliar os efeitos da agressividade fiscal praticada pelas empresas sobre o seu investimento em diferentes pontos da distribuição amostral. A técnica utilizada mostrouse particularmente adequada para se lidar com a presença de outliers e a anormalidade dos resíduos. Resultados/Discussão: Os resultados demonstram que há uma relação estatística significativa entre agressividade fiscal e investimento, em particular em relação a variável Taxa Efetiva de Tributação (GAAPETR), o que sugere que empresas que praticam a agressividade fiscal estão associadas positivamente a um maior nível de investimentos.

Contribuições: $\mathrm{O}$ artigo contribui para a literatura de agressividade fiscal na medida em que apresenta evidências de que as sobras de caixa geradas pelo planejamento tributário contribuem para o aumento dos níveis de investimentos das empresas.

Palavras-chave: Agressividade fiscal; Investimentos; Planejamento tributário.

${ }_{1}^{1}$ rodofama2@gmail.com. Universidade Federal do Espírito Santo. Vitória-ES, Brasil. https://orcid.org/0000-0001-9802-8332

${ }^{2}$ eduardozanoteli@gmail.com. Universidade Federal do Espírito Santo. Vitória-ES, Brasil. https://orcid.org/0000-0001-9842-7990

- DOI: http://dx.doi.org/10.14392/asaa.2020130304

- Artigo submetido em: 10/05/2020. Aceito em: 26/10/2020. 


\section{FISCAL AGGRESSIVITY AND INVESTMENTS IN THE BRAZILIAN STOCK MARKET}

Abstract:

Objective: Based on the assumption that the benefits of tax planning are generated in the form of financial reserves arising from the reduction of tax expenditures, the aim of this paper is to analyze the relationship between tax aggressiveness practices and corporate investment.

Method: The sample consists of 2,752 observations of public companies in 13 sectors. The data collected is from 2010 to 2018. Through the Quantile Regression, the paper evaluates the effects of tax aggressiveness practiced by companies on their investment of the sample. The technique used is particularly suitable for dealing with the presence of outliers since the residues does not follow a normal distribution.

Results/Discussion: The results demonstrate that there is a significant relationship between tax aggressiveness and investment, specifically with Effective Tax Rate (GAAPETR), which suggest that companies that use tax aggressiveness practices are positively associated with a higher level of investments.

Contributions: The paper contributes to the literature of tax aggressiveness since it presents evidence that the cash generated in excess by tax planning contributes to the increase in investment by companies.

Keywords: Tax aggressiveness; Investments; Tax avoidance. 


\section{INTRODUÇÃO}

$A^{\text {sas }}$ partir de casos amplamente divulgados pela imprensa, e que representaram grandes escândalos de firmas que manipulavam os seus relatórios financeiros, o interesse da academia para com o tema agressividade fiscal aumentou consideravelmente. Como exemplo, cita-se o caso da multinacional Starbucks, que reportou perdas em seus relatórios contábeis no Reino Unido com o intuito de diminuir sua taxa de imposto efetivo pagos no país, apesar de deter aproximadamente um terço do percentual de mercado (Kleinbard, 2013).

Os benefícios da agressividade fiscal dentro de uma empresa são gerados na forma de reservas financeiras provenientes de reduções nos gastos tributários (Park et al., 2016). Esta prática é considerada um trade-off entre ganhar, se não for detectada, e perder, caso contrário (Slemrod, 2004). Além das distorções que impedem os mercados de operar perfeitamente, como tributação e custos de transação, têm-se os problemas de agência e informação assimétrica, que afetam a eficiência do investimento (Stein, 2003).

Conforme Hanlon e Slemrod (2009), o mercado reage positivamente com a divulgação dos seus respectivos relatórios financeiros que evidenciam esforços para reduzir os tributos pagos. Por isso, pode-se dizer que a transparência contábil pode ter efeitos tanto benéficos (aumentar o fluxo de caixa, e.g.) quanto maléficos (danos à imagem da empresa, multas fiscais, e.g.).

As pesquisas no Brasil relacionadas à agressividade fiscal têm destacado os seguintes aspectos: empresas familiares (Martinez \& Ramalho, 2014); seus efeitos no valor da companhia (Santana \& Rezende, 2016); seus impactos no custo da dívida (Da Silva \& Martinez, 2017); comparação de empresas da NYSE (The New York Stock Exchange) com as da [B] ${ }^{3}$ (Araújo, Santo, Leite Filho \& Camara, 2018); refazimento das Demonstrações Financeiras (Ramos \& Martinez, 2018) e eficiência operacional (Junior \& Martinez, 2018).

Já a literatura internacional tem analisado temas como: incentivos contratuais (Desai \& Dharmapala, 2006); cultura corporativa (Frank, Lynch \& Rego, 2009); qualidade da informação contábil (Biddle \& Hilary, 2006); Biddle, Hilary \& Verdi, 2009); e os efeitos na eficiência do investimento (Goldman, 2016).

Observa-se a escassez de estudos que buscam ampliar a compreensão do impacto do planejamento tributário sobre o investimento das empresas, embora alguns trabalhos mereçam destaque, dentre eles: Mayberry (2012); Blouin, Devereux e Shackelford (2012); Cheng, Dhaliwal e Zhang (2013); Blaylock (2016); Goldman (2016); Junior e Martinez (2018); e Alencastre, Campos e Marques (2018). Nesse sentido, entender o que torna um investimento eficiente é fundamental para relacioná-lo com o planejamento tributário.

Outro aspecto importante é a qualidade da informação contábil que tem efeito crucial na definição do nível da agressividade fiscal. Biddle e Hilary (2006) constataram que o vínculo entre os fluxos de caixa gerados internamente e o investimento é mais fraco quando a qualidade contábil é alta, portanto, um maior monitoramento mitiga o planejamento tributário agressivo. Biddle, Hilary e Verdi (2009) e Cheng, Dhaliwal e Zhang (2013) concluíram que a divulgação de informações contábeis tende a mitigar assimetrias informacionais que dificultam o investimento eficiente.

Blaylock (2016) supôs que os administradores obscurecem os relatórios contábeis no intuito de tornar mais difícil para as autoridades tributárias identificarem a agressividade fiscal. Ele concluiu que a agressividade fiscal geralmente está associada positivamente à performance futura da empresa e, ainda, que não há consistência de que o Planejamento Tributário cause investimento ineficiente quando a governança das companhias é fraca. 
Por outro lado, Mayberry (2012) examinou a relação entre a evasão fiscal e o investimento da companhia recorrendo à seleção adversa e ao risco moral para fornecer uma ligação entre a evasão fiscal e o investimento. $\mathrm{O}$ autor concluiu que as empresas confiam nas economias tributárias provenientes da evasão fiscal para aumentar os recursos de caixa, o que aumenta o valor da empresa ao canalizar os investimentos para projetos com valor presente positivo.

Blouin, Devereux e Shackelford (2012) descobriram que a agressividade fiscal aumenta o nível de investimento da empresa em âmbito internacional. De acordo com esses autores, as UTBs (uncertain tax benefits), consideradas como reservas financeiras da companhia que diminuem o valor em imposto a ser pago, tratam-se de uma prática incerta, por isso seu valor aumenta até o ponto que a empresa calcula que seus benefícios marginais são maiores que seus possíveis custos.

Goldman (2016) analisou os aspectos de empresas com acesso a fundos de investimento; nelas, o planejamento tributário agressivo está associado a níveis mais altos de investimentos. Além disso, o planejamento agressivo está associado a altos custos de agência no que tange à tomada de decisão dos administradores em relação aos fluxos de caixa livres da empresa, ao reduzir a qualidade da informação contábil.

Ainda com relação à eficiência do investimento, Junior e Martinez (2018, p. 2) investigaram se empresas menos eficientes em termos operacionais se engajam em estratégias de agressividade fiscal com mais frequência. Os resultados ratificaram a hipótese proposta e eles concluíram que a capacidade das empresas de utilizar com eficiência seus recursos têm um impacto significativo no planejamento tributário.

Resultado que não foi corroborado por Alencastre, Campos e Marques (2018), os quais constataram que empresas mais agressivas tributariamente apresentam maior eficiência produtiva. Os autores concluíram ainda que a variável ETR pode não ser a mais adequada para se calcular a agressividade fiscal, uma vez que não apresentou significância estatística no seu estudo.

Ante o exposto, observa-se a necessidade de se aprofundar a compreensão do impacto da agressividade fiscal sobre os investimentos das empresas, donde decorre a seguinte questão de pesquisa: $\mathrm{O}$ planejamento tributário agressivo impacta o investimento das empresas? O objetivo geral da presente pesquisa é analisar a relação das práticas de agressividade fiscal com o investimento das empresas.

O tema é relevante, uma vez que, para a economia, um aumento de $1 \%$ da carga tributária total tende a reduzir o PIB em cerca de 0,3\% no Brasil (Sachsida, Mendonca \& Moreira, 2016, p. 23). Além disso, a estrutura tributária brasileira é ineficiente, posto que privilegia os tributos sobre o consumo, em vez de sobre a renda e o lucro, que trariam mais equidade na proporção do pagamento dos impostos. Portanto, a pesquisa fiscal tem relevância tanto para os órgãos reguladores quanto para investidores, acadêmicos, analistas, tributaristas, contadores e empresários.

Além da Introdução, são apresentadas outras quatro seções. Na seção 2 é apresentada e discutida a literatura relevante sobre o tema, a qual suporta a Metodologia utilizada (seção 3) e as análises dos resultados (seção 4). Com base nas seções 2, 3 e 4 são apresentadas na seção 5 as Considerações Finais.

\section{REVISÃO DA LITERATURA}

Allingham e Sandmo (1972) examinaram alguns aspectos relacionados à decisão de evasão de tributos, com isso, iniciou-se uma nova fase de estudos dentro da pesquisa tributária, ao analisar a escolha do contribuinte individual em evadir impostos. Nesta pesquisa, sugeria-se que as taxas tributárias em si eram o instrumento disponível para neutralizar a tendência à evasão, assim como a aplicação de penalidades da lei e o aumento da fiscalização tributária. 
Posteriormente, Scholes e Wolfson (1990) propuseram o paradigma all taxes, all costs, all parts, que inovou ao tratar o planejamento tributário de forma interdisciplinar. Com isso, o fato de que um planejamento tributário ineficiente gera custos às empresas (Goldman, 2016; Martinez \& Silva, 2017; Junior \& Martinez, 2018) ficou mais latente e amparou teoricamente muitos trabalhos relacionados à pesquisa fiscal.

Já Shackelford e Shevlin (2001) fizeram uma revisão de literatura e exploraram os trade-offs que os gerentes enfrentam entre decisões financeiras e fiscais, ao analisar considerações regulatórias e a destinação de recursos para abrigos fiscais. Os autores salientam que as pesquisas fiscais existentes podem ser pensadas como uma tentativa de entender os trade-offs e significados econômicos de fatores tributários e não tributários nas decisões de negócios, pois quanto mais os tributos afetam a tomada de decisão, mais eles reduzem a eficiência econômica.

\subsection{Planejamento Tributário e Agressividade Fiscal}

O modelo Scholes-Wolfson enfatiza os impactos nas decisões tributárias, a qual é mais bem detalhada a seguir (Scholes et al., 2015, p. 1):

\footnotetext{
[...] todas as partes (1) que, quando se executa um planejamento tributário agressivo, deve-se analisar seus efeitos na arrecadação governamental, nos empregados, na empresa e nos consumidores, ou seja, uma perspectiva contratual multilateral. Com relação ao paradigma todos os impostos (2), esse se refere a toda carga tributária incidida nas operações da companhia, variando de um setor para outro, e do regime fiscal envolvido, ou seja, a importância das considerações fiscais nos ajustes de preços. Por último, pode-se dizer que o paradigma todos os custos (3) se refere tanto aos custos explícitos (Custos de auditoria, sistemas de informação e despesas trabalhistas, por exemplo) e implícitos de operação da companhia, o que significa que compreender tributos exige entender fatores não-tributários.
}

Shackelford Shevlin (2001) ressaltam, ainda, que fatores não tributários também devem ser considerados no desenvolvimento de estratégias tributárias, como incentivos financeiros e os custos de transação, e.g. Os autores alertam que, como o modelo Scholes-Wolfson opera com hipóteses, qualquer descoberta pode ser caracterizada como consistente com o modelo, pois os custos não tributários são difíceis de quantificar.

Existem duas correntes teóricas que afirmam que a agressividade fiscal impacta o valor da empresa. Uma é a corrente tradicional, a qual afirma que não há custos associados às práticas de agressividade fiscal, por isso todas as empresas a praticam (Park, Ko, Jung \& Lee, 2016), enquanto a outra é a teoria da agência. Nesta, as divergências de interesses entre os administradores e os acionistas são tratadas como conflitos de agência, os quais geram custos adicionais para as empresas advindos tanto do risco moral quanto da seleção adversa (Blanchard, Lopezde-Siianesb \& Shleifet, 1994).

A teoria de agência considera os custos não tributários, como: os necessários ao planejamento tributário, a elaboração dos relatórios financeiros, problema de agência, custos políticos e os relativos à reputação da empresa (Park et al., 2016), que podem levá-la a incorrer em práticas ilegais. Na visão de Lisowsky, Robinson e Schmidt (2010), a agressividade fiscal é um conjunto de ações no fim da escala de legalidade, isto é, de cunho evasivo, as quais variam desde o planejamento tributário legítimo até investimentos abusivos em paraísos fiscais, que têm o intuito de criar incerteza (Lisowsky et al., 2010).

Evasão fiscal, agressividade fiscal e abrigos fiscais estão todos relacionados com essas considerações, pois constituem diferentes formas de maximizar o lucro após o pagamento de tributos (Lietz, 2013). Ao explicar melhor o modelo Scholes-Wolfson, Shackelford e Shevlin (2001, p. 323) dão exemplos: 
[...] todas as partes: as estruturações salariais, que têm efeitos tanto na lucratividade da companhia quanto na remuneração salarial. Quanto a todos os impostos, pode-se citar a escolha de investir em um ativo com um retorno menor por ter isenção fiscal, dando ganho de rendimento líquido relativamente maior, ao considerar os fatores tributários. Em relação a "todos os custos", este seria o trade-off entre seguir a legislação tributária ou minimizá-la para atingir os objetivos fiscais, a depender da estratégia da empresa.

Com isso, a agressividade fiscal aumenta a incerteza da lucratividade futura da empresa, o que intensifica a assimetria informacional e, consequentemente, afeta de forma negativa as expectativas do mercado (Park et al., 2016). Além disso, o risco de caixa aumenta a partir das transações relacionadas a essas estratégias fiscais, como operações estrangeiras, atividades de pesquisa e desenvolvimento e investimentos em intangíveis (Goh, Lee, Lim \& Shevlin, 2016).

As empresas, portanto, empregam estratégias fiscais com o objetivo de melhorar os resultados contábeis (Desai \& Dharmapala, 2006). Os departamentos fiscais são muitas vezes operados como centros de lucro (Robinson, Sikes \& Weaver, 2010), uma vez que, quanto menos se pagam tributos, maior o excedente de caixa.

Por vezes, abrigos fiscais (tax shelters) tornam-se escolhas lógicas, pois englobam várias legislações de tributos diferentes, o que possibilita à companhia escolher o mais adequado aos seus objetivos. Nesse sentido, Frank et al. (2009) definem relatórios financeiros como agressivos quando se visa aumentar o caixa dentro ou fora da legalidade, bem como consideram relatórios fiscais agressivos como uma manipulação descendente do lucro tributável através do planejamento tributário que pode ou não ser evasão fiscal fraudulenta.

Os abrigos fiscais criam economias tributárias ao recondicionar os direitos de propriedade entre os investidores. Historicamente, eles foram distinguidos pela dedutibilidade de um investimento a uma taxa que excede sua depreciação econômica (Scholes \& Wolfson, 1992). O agente oportunista escolhe a opção mais vantajosa para desviar o recurso (Desai \& Dharmapala, 2006).

As diferenciações legais entre os termos relacionados às práticas fiscais consideram o aspecto da ocorrência do fato gerador e da licitude da ocorrência. Quanto à diferença entre sonegação e elisão fiscal, Torres $(2013$, p. 7) esclarece que:

De maneira legal, a diferença entre sonegação e elisão fiscal refere-se à licitude, sendo aquela sempre ilícita. Esta pode ser tanto lícita quanto ilícita, diferindo o planejamento legítimo (elisão lícita) com o planejamento abusivo (elisão abusiva) [...] a evasão lícita e a elisão (lícita ou ilícita) precedem a ocorrência do fato gerador no mundo fenomênico. A sonegação e a fraude (evasão ilícita) dão-se após a ocorrência daquele fato.

A Figura 1 a seguir ajuda a visualizar o gradiente entre o planejamento tributário legal (elisão) e ilegal (evasão) e o grau de agressividade fiscal (tributária):

Figura 1 - Escala de legalidade do planejamento tributário

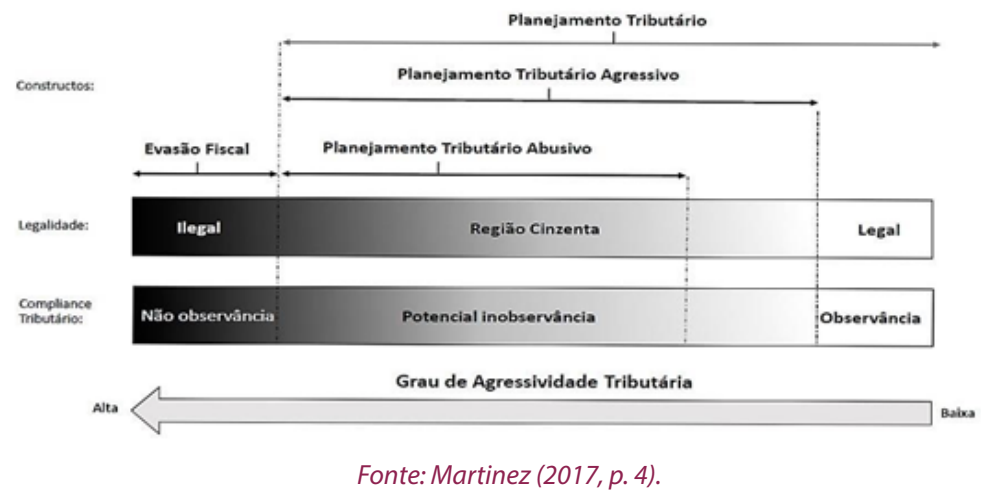


Observe-se que os esforços efetuados para reduzir o ônus da tributação pelo sujeito passivo podem ser elencados nos seguintes perfis: o conservador, aquele que evita pagar mais para não ser multado; o moderado, que demonstra aspectos arrojados ao procurar brechas na lei; e, por último, o arriscado, que exibe características do perfil moderado com viés de uma interpretação pró-contribuinte (aqui, a agressividade fiscal se aproxima da evasão e, por vezes, chega às vias de fato).

Crabtree e Kubick (2014) estudaram o quanto a agressividade fiscal afeta a precisão da divulgação dos resultados contábeis. Isso, conforme os autores, traz como consequência o fato de que a evasão fiscal se torna uma característica de risco significativa para as empresas e que deve ser considerada na avaliação de valor de mercado da empresa em questão, assim como tem se tornado uma prática recorrente no mercado financeiro, acentuando o risco de agência e a informação assimétrica.

Ao estudar as práticas das empresas, Graham, Hanlon, Shevlin e Shroff (2014) investigaram os incentivos que os gerentes das empresas evitam incorrer em práticas de tax avoidance ao enviar questionários de amostra aos gerentes. Eles constataram a falta de finalidade financeira neste tipo de transação e os possíveis custos à reputação da empresa, uma vez que a atenção negativa atraída pela mídia é um fator desestimulante das práticas de planejamento tributário, como também observou Zimmerman (1983).

Martinez e Ramalho (2014) investigaram a intensidade em termos de planejamento tributário de empresas não familiares e familiares no Brasil. Para a variável BTD (Book tax Difference), as empresas familiares apresentaram um sinal positivo, indicando uma tendência para maior BTD. Por sua vez, a ETR teve um sinal negativo, identificando que as empresas familiares são mais agressivas. Já Araújo et al. (2018) compararam a agressividade fiscal das empresas na NYSE com as da $[B]^{3}$ e concluíram que as empresas da NYSE possuem maior qualidade contábil e, portanto, praticam menos planejamento tributário agressivo.

\subsection{Decisão de Investimentos e Planejamento Tributário}

Quando os gerentes têm acesso a um fluxo de caixa elevado, em teoria o investimento converge para o nível ótimo. No entanto, quando os administradores enfrentam restrições de caixa, haverá pouco espaço para o crescimento da empresa, pois há custos associados ao financiamento externo (Caldeira \& Loncan, 2013). Nesse sentido, Stein (2003, p. 121) elencou motivos que geram os conflitos de interesse capazes de afetar o investimento da empresa, dentre os quais estão:

[...] construção de poder (empire building) ${ }^{2}$, autopreservação e diversificação, preocupação quanto à reputação da carreira, visão de curto prazo, necessidade de estar em grupo (herding), uma vida sem stress (quiet life), consequências da tomada de decisões, confiança excessiva e decisões de investimentos distorcidas dos fundamentos do mercado.

Além disso, uma vez esgotadas as estratégias fiscais, a companhia tende a recorrer a mecanismos de financiamento externos para manutenção de suas atividades. Dessa forma, os fatores que determinam as relações entre as decisões de investimento, o planejamento tributário e consequentemente a estrutura de capital são cruciais para a sobrevivência da empresa. Por outro lado, a legislação tributária influencia as decisões de financiamento das empresas por meio do seu efeito sobre o custo de capital.

Scholes, Wilson e Wolfson (1990) encontraram adequação do portfólio do setor bancário devido a mudanças de política de dedutibilidade de juros, assim como uma relação entre as decisões de investimento e suas taxas marginais de tributação efetiva, uma vez que os bancos estão mais inclinados ao planejamento tributário agressivo quando os custos são menores do que o benefício fiscal (Scholes et al., 1990). 
Segundo Jensen e Meckling (1994), entender os incentivos do comportamento humano é fundamental para compreender as decisões que envolvem uma organização. Os problemas entre o agente e o principal surgem porque os gerentes obtêm vantagens em detrimento da organização, como a prática do empire building (aumentar sua importância e autoridade) e maximização da sua quota no mercado.

Robinson et al. (2010) investigaram por que as empresas optam por avaliar seu departamento tributário como um centro de lucro em vez usá-lo como custeio das suas atividades, assim como a associação entre essa escolha e o nível da tributação efetiva. Os autores constataram que empresas maiores, diversificadas e que possuem mais oportunidades de planejamento tributário em relação às unidades de negócios têm mais probabilidade de considerar os departamentos fiscais como centros de lucro e que o planejamento tributário tem efeitos positivos nos lucros reportados, o que explica por que as empresas fornecem incentivos aos executivos para reduzir os encargos tributários.

Almeida e Campello (2007) tentaram identificar se o fato de a companhia poder ceder um ativo em garantia afeta suas decisões de investimento. Os autores classificaram como empresas sem restrições financeiras aquelas cujos ativos são avaliados por uma agência classificadora de risco e descobriram que, embora a tangibilidade dos ativos para companhias sem restrição financeira esteja relacionada a um menor custo de capital, esses efeitos não são observados em empresas sem restrições financeiras, pois as que possuem maior tangibilidade estão menos propensas a ter tais limitações de caixa.

Como forma de se obter maior retorno ao investimento, algumas empresas optam por utilizar financiamento em suas atividades, em vez de capital próprio. Conforme estudo de Martinez e Silva (2017, p. 240), "as empresas menos agressivas arcam com custo da dívida maior, pois os credores demandam um custo de captação dos recursos proporcional ao risco antecipado". Os autores salientam que os variados métodos de planejamento tributário disponíveis no Brasil podem causar confusão nos analistas — ou seja, efeito controverso.

Determinar o mecanismo de financiamento é fundamental para a sobrevivência da companhia. Balakrishnan, Core e Verdi (2014) investigaram a relação entre a qualidade do relatório contábil e o financiamento e o investimento da empresa, assim como as modificações desses relatórios quanto à mudança na capacidade de financiamento e constataram que há menos alteração na capacidade de financiamento e investimento para companhias que têm recursos internos de controle mais bem estabelecidos.

Se por um lado a agressividade fiscal pode ser uma fonte importante de fluxo de caixa para aliviar as restrições de capital da empresa (Mayberry, 2012), por outro, a evasão fiscal pode aumentar substancialmente a variância dos fluxos de caixa da empresa (Goh et al., 2016), o que torna o investimento mais arriscado. Portanto, considerar todas as partes envolvidas nas atividades de uma empresa envolve o direcionamento dos recursos provenientes das práticas de agressividade fiscal e seu efeito no de investimento.

Ante o exposto postula-se a seguinte hipótese de pesquisa:

H1: Empresas que praticam a agressividade fiscal estão associadas positivamente a um maior investimento.

Desse modo, testam-se se os efeitos do planejamento tributário agressivo no investimento, na medida em que existe correlação entre a agressividade fiscal e as ações gerenciais (Desai \& Dharmapala, 2006). Dado que os detentores de capital estão expostos ao risco de desvio dos recursos por parte dos administradores das empresas, sendo este um problema de agência com relação à destinação dos recursos, não necessariamente tendo efeito positivo no investimento. 


\section{METODOLOGIA}

A presente pesquisa tem natureza descritiva e adotou uma abordagem quantitativa por mostrar-se alinhada ao seu objetivo e por permitir testar a hipótese postulada. No intuito de se buscar uma técnica com robustez frente à presença de outliers, foi adotada a regressão quantílica. As vantagens quanto a esse tipo de regressão apontadas por Koenker e Bassett (1978) são: ela é sugerida quando a distribuição não é gaussiana (normal); é robusta a outliers; quando os resíduos não são normais e/ou não homocedásticos produzem estimadores mais eficientes do que os da regressão por mínimos quadrados ordinários (MQO); e é mais informativa, não só ficando restrita a uma média, pois pode-se obter a regressão nos quantis definidos (25\%, 50\% e $75 \%)$.

De acordo com a regressão quantílica, avaliaram-se os efeitos da agressividade fiscal praticada pelas empresas sobre o investimento destas em diferentes pontos da distribuição. A amostra foi obtida a partir dos dados relativos aos exercícios de 2010 a 2018 de empresas listadas na [B] $]^{3}$, e do ano de 2009 para variáveis defasadas, menos o setor financeiro, que possui regime de tributação diferenciado, excluindo-se ainda as empresas que não possuíam informações disponíveis para as variáveis de controle.

Os dados foram coletados em relação à sua frequência anual. Utilizou-se como base de coleta dos dados a plataforma Comdinheiro. Consultas aos sites oficiais das próprias empresas foram efetuadas como forma de completar dados faltantes para as variáveis independentes, apenas. Visto isso, o modelo 1 proposto é utilizado como o padrão para os outros que foram testados através da regressão a seguir:

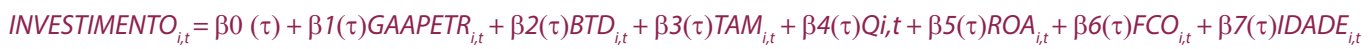
$+\beta 8(\tau) Z_{i, t}+\beta 9(\tau) T_{C R}+\beta 10(\tau) \operatorname{TAN}_{i, t}+\beta 11(\tau) E_{i, t}+\beta 12(\tau) D_{i, t}$ ummy $_{i, t}+\beta 13(\tau)$ DummySCP $_{i, t+\varepsilon i}$

Para dar maior robustez ao modelo, foi criada uma dummy com as medianas dos setores utilizados na amostra. Optou-se pela utilização desta para o cálculo das dummies, uma vez que, segundo Theóphilo e Martins (2009), esta é o valor que divide a amostra em duas partes iguais. Com isso, obtiveram-se dados para o cálculo de mais quatro modelos. Um utilizando as Dummies GAAPETR e BTD, outro apenas a Dummy GAAPETR, assim como mais um para a Dummy BTD e, por último, a interação das dummies GAAPETR e BTD, totalizando 8 regressões.

No Quadro 1 a seguir são apresentadas as variáveis dos modelos, sua metodologia de cálculo, sinal esperado e tipologia bem como a literatura que as justifica e fundamenta:

Quadro 1 - Especificação das variáveis dos modelos

\begin{tabular}{|c|c|c|c|c|c|}
\hline Referências & Tipo de Variável & Sinal & Variável & Fórmula & Definição \\
\hline Goldman (2016) & Dependente & NA & INVESTIMENTO & $\frac{\Delta \text { Imobi,t - Depre. }}{\text { ATi,t }}$ & $\begin{array}{c}\text { Nível do investimento da } \\
\text { empresa }\end{array}$ \\
\hline $\begin{array}{l}\text { Hanlon e Heitzman } \\
\text { (2010) }\end{array}$ & \multirow{2}{*}{ Independente } & + & GAAPETR & $\frac{\mathrm{IR}_{\mathrm{it}, \mathrm{CSLL}}+\mathrm{CSL}_{\mathrm{i,t}}}{-\mathrm{LAIR}_{\mathrm{i}, \mathrm{t}}}$ & Taxa de Tributação Efetiva \\
\hline $\begin{array}{l}\text { Frank, Lynch e Rego } \\
\text { (2009) }\end{array}$ & & + & BTD & LAIR $_{\mathrm{i}, \mathrm{t}}-\frac{\mathrm{IR}_{\mathrm{i}, \mathrm{t}}+\mathrm{CSLL}_{\mathrm{i}, \mathrm{t}}}{-0,34}$ & Book tax Difference \\
\hline Elaborado pelo autor & Dummy Independente & + & $\begin{array}{l}\text { DUMMYGAAPETR e } \\
\text { DUMMYBTD }\end{array}$ & $1 ; 0$ & $\begin{array}{l}1 \text { se a variável é maior do } \\
\text { que a mediana do setor, } 0 \\
\text { caso contrário }\end{array}$ \\
\hline
\end{tabular}




\begin{tabular}{|c|c|c|c|c|c|}
\hline $\begin{array}{l}\text { Cheng, Dhaliwal, } \\
\text { Zhang (2013); Biddle, } \\
\text { Hilary, Verdi (2009) }\end{array}$ & \multirow{11}{*}{ Controle } & - & TAM & $\log _{n} A T$ & $\begin{array}{l}\text { Logaritmo natural do ativo } \\
\text { total no ano corrente }\end{array}$ \\
\hline $\begin{array}{l}\text { Desai e Dharmapala } \\
\text { (2009) }\end{array}$ & & + & Q & $\frac{(\mathrm{DL}+\mathrm{VM})_{\mathrm{i}, \mathrm{t}}}{-\mathrm{AT}_{\mathrm{i}, \mathrm{t}}}$ & $\begin{array}{l}\text { Oportunidades de } \\
\text { investimento da empresa }\end{array}$ \\
\hline $\begin{array}{l}\text { Frank, Lynch e Rego } \\
\text { (2009) }\end{array}$ & & + & ROA & $\frac{\mathrm{EBIT}_{\mathrm{i}, \mathrm{t}}}{\mathrm{AT}_{\mathrm{i}, \mathrm{t}-1}}$ & $\begin{array}{c}\text { Retorno Operacional da } \\
\text { Empresa }\end{array}$ \\
\hline Goldman (2016) & & - & FCO & $\begin{array}{l}\mathrm{FCO} \\
\mathrm{AT}_{\mathrm{i}, \mathrm{t}-\mathrm{I}}\end{array}$ & Fluxo de Caixa Operacional \\
\hline $\begin{array}{l}\text { Cheng, Dhaliwal e } \\
\text { Zhang (2013); Biddle, } \\
\text { Hilary e Verdi (2009) }\end{array}$ & & - & IDADE & $\log _{n} A$ nos & $\begin{array}{c}\text { Logaritmo natural do tempo } \\
\text { de registro na }[\mathrm{B}]^{3} \text { até a } \\
\text { data atual }\end{array}$ \\
\hline Altman (1968) & & + & z & $\begin{array}{c}1.2^{*} \mathrm{~A}+1.4^{*} \mathrm{~B}+3.3^{*} \mathrm{C} \\
+0.6^{*} \mathrm{D}+\mathrm{E}\end{array}$ & $\begin{array}{c}\text { Proxy para probabilidade } \\
\text { de falência financeira da } \\
\text { empresa }\end{array}$ \\
\hline Goldman (2016) & & + & TCR & $\frac{\mathrm{RL}_{\mathrm{i}, \mathrm{t}}-\mathrm{RL}_{\mathrm{i}, \mathrm{t}-1}}{-\mathrm{RL}}$ & $\begin{array}{l}\text { Taxa de Crescimento da } \\
\text { Receita da empresa }\end{array}$ \\
\hline $\begin{array}{l}\text { Almeida e Campello } \\
\text { (2007) }\end{array}$ & & - & TAN & $\frac{\mathrm{IMOB}_{\mathrm{it}, \mathrm{t}}}{-\mathrm{AT}_{\mathrm{i}, \mathrm{t}}}$ & Tangibilidade dos ativos \\
\hline $\begin{array}{l}\text { Myers (1977); Almeida } \\
\text { e Campello (2007) }\end{array}$ & & - & END & $\frac{P C_{i, t}+P N C_{i, t}}{A T_{i, t}}$ & Nível de Endividamento \\
\hline \multirow{2}{*}{$\begin{array}{l}\text { Cheng; Dhaliwal e } \\
\text { Zhang (2013); Biddle, } \\
\text { Hilary, e Verdi (2009) }\end{array}$} & & & DUMMYDIV & \multirow[b]{2}{*}{$1 ; 0$} & \multirow{2}{*}{$\begin{array}{l}\text { Assume o valor } 1 \mathrm{se} \mathrm{a} \\
\text { empresa paga Juros Sobre o } \\
\text { Capital Próprio/dividendos, } 0 \\
\text { caso contrário }\end{array}$} \\
\hline & & - & DUMMYJSCP & & \\
\hline
\end{tabular}

Fonte: Elaborado pelos autores.

O modelo utilizado foi adaptado de Goldman (2016), visto que no modelo original foram utilizadas as variáveis DTAX e DiffETR para o cálculo da agressividade fiscal e na presente pesquisa utiliza-se a taxa tributária efetiva sobre o lucro contábil (GaapETR) e a diferença entre o lucro contábil e tributário (BTD), variáveis que permitem observar distorções na base de cálculo do Imposto de Renda Pessoa Jurídica (IRPJ) e da Contribuição Social sobre o Lucro Líquido (CSLL), além de ampliar as variáveis de controle no sentido de aprofundar a compreensão sobre o impacto da agressividade fiscal sobre os investimentos das empresas.

Buscou-se avaliar a maturidade das empresas por meio das variáveis tamanho, idade e fluxo de caixa; enquanto as oportunidades de crescimento foram avaliadas por meio das variáveis retorno sobre os ativos (ROA), Q de Tobin (1969), tangibilidade, endividamento, bem como pela taxa de crescimento da receita. Por último, as variáveis dummy de dividendos e de juros sobre o capital próprio foram incluídas, pois representam parte do retorno sobre o investimento dos acionistas.

\section{APRESENTAÇÃO E ANÁLISE DOS DADOS E RESULTADOS}

Em princípio, as variáveis na regressão adotada englobaram os seguintes setores (13) e empresas (4.764): Bens Industriais (1.022), Construção e Transporte (45), Consumo Cíclico (1.022), Consumo Não Cíclico (365), Hotéis e Restaurantes (3), Materiais Básicos (639), Não Classificado (5), Petróleo, Gás e Combustível (140), Químicos (2), Saúde (187), Tecnologia da Informação (55), Telecomunicações (58) e Utilidade Pública (951). Após a exclusão de variáveis sem dados ou com dados incompletos a amostra se consolidou com 2.752 observações.

4.1 Regressões Quantílicas

A partir do modelo (1) proposto para a hipótese de que as empresas que praticam a agressividade fiscal estão associadas positivamente a um maior investimento, os outros modelos seguiram derivações 
na forma de cálculo em relação às variáveis independentes, apenas. Com o objetivo de simplificar a explanação das regressões realizadas apresenta-se na Tabela 1 a seguir as variáveis independentes de cada modelo:

Tabela 1 - Modelos propostos
\begin{tabular}{|c|c|c|}
\hline Modelo & \multicolumn{2}{|c|}{ Variáveis independentes } \\
\hline 1 & GAAPETR & BTD \\
\hline 2 & GAAPETR & - \\
\hline 3 & - & BTD \\
\hline 4 & \multicolumn{2}{|c|}{ GAAPETR*BTD } \\
\hline 5 & DUMMYGAPETR & DUMMYBTD \\
\hline 6 & DUMMYGAAPETR & - \\
\hline 7 & - & DUMMYBTD \\
\hline 8 & DUMMYGAAPETR*DUMMYBTD \\
\hline
\end{tabular}

Fonte: Elaborado pelos autores.

Dessa forma, analisa-se o efeito de cada variável independente de forma isolada no intuito de capturar qual tem maior relevância com relação a seus impactos no investimento e seus efeitos nas varáveis de controle. Os modelos 4 e 8 representam a interação (multiplicação) das variáveis independentes, caracterizando um dos aspectos diferenciais desta pesquisa. Devido a presença de outliers, assim como os erros da regressão linear não assumiram algumas premissas exigidas, optou-se pela aplicação da Regressão Quantílica, pois estima melhor a associação das variáveis a partir da mediana, além de verificar diversos pontos de cortes nos valores do investimento ao invés de apenas a média.

Foram testadas diversas regressões, em que não foi possível encontrar uma que satisfizesse a premissa de resíduos normais - entre elas, o modelo com retirada de variáveis (forward), com transformação na variável dependente, com o método de winsorização, por mínimos quadrados generalizados, e nenhuma obteve êxito na premissa. Assim, com a finalidade de explorar os dados com as suas características originais e manter o maior número de observações possíveis a Regressão Quantílica foi utilizada.

\subsection{Resultados}

Observando a princípio as variáveis quantitativas, é possível perceber uma grande variabilidade em todas as variáveis, sendo as de menor variabilidade em relação à média: a de Tamanho (TAM) e a idade da firma (IDADE). As demais variáveis apresentaram desvios-padrões bastante elevados, o que indica que há outliers presentes na amostra. Nas categóricas, apenas a Dummy GAAPETR apresentou porcentagem maior para a categoria 1, ou seja, a medida de agressividade fiscal foi maior que a divisão mediana do setor (Tabela 2). 
Tabela 2: Estatística descritiva das variáveis quantitativas

\begin{tabular}{|c|c|c|c|c|c|c|c|}
\hline & & $\mathrm{n}$ & Mínimo & Máximo & Mediana & Média & Desvio-padrão \\
\hline INVESTIMENTO & & 2752 & $-801,78$ & 121,95 & $-0,01$ & $-0,26$ & 15,88 \\
\hline GAAPETR & & 2752 & $-983,24$ & 947,92 & 0,15 & 9,57 & 180,81 \\
\hline BTD & & 2752 & $-84052,60$ & 48137,40 & $-157,93$ & $-448,43$ & 4241,36 \\
\hline TAM & & 2752 & 5,23 & 999,84 & 690,24 & 599,99 & 297,83 \\
\hline Q & & 2752 & $-0,45$ & 1579,41 & 0,60 & 36,53 & 100,79 \\
\hline ROA & & 2752 & $-885,48$ & 654,73 & 0,07 & 2,33 & 50,44 \\
\hline $\mathrm{FCO}$ & & 2752 & $-552,84$ & 751,72 & 0,06 & 2,78 & 42,01 \\
\hline IDADE & & 2752 & 0,00 & 477,91 & 309,10 & 285,28 & 110,11 \\
\hline z & & 2752 & $-961,31$ & 1531,81 & 115,11 & 110,60 & 173,04 \\
\hline TCR & & 2752 & $-234,72$ & 1279,31 & 0,10 & 10,21 & 74,90 \\
\hline TAN & & 2752 & 0,00 & 129,96 & 0,29 & 0,49 & 4,76 \\
\hline END & & 2752 & 0,07 & 938,53 & 0,63 & 23,47 & 79,53 \\
\hline GAAPETR X BTD & & $\begin{array}{c}2751 \\
n\end{array}$ & $\begin{array}{c}-167660,00 \\
\%\end{array}$ & 1300000,00 & 11,84 & 1499,41 & 44329,50 \\
\hline \multirow{2}{*}{ DUMMYDIV } & 0 & 1832 & 66,57 & - & - & - & - \\
\hline & 1 & 920 & 33,43 & - & - & - & - \\
\hline \multirow{2}{*}{ DUMMYJSCP } & 0 & 2120 & 77,03 & - & - & - & - \\
\hline & 1 & 632 & 22,97 & - & - & - & - \\
\hline \multirow{2}{*}{ DUMMYGAAPETR } & 0 & 1730 & 62,86 & - & - & - & - \\
\hline & 1 & 1022 & 37,14 & - & - & - & - \\
\hline \multirow{2}{*}{ DUMMYBTD } & 0 & 1835 & 66,68 & - & - & - & - \\
\hline & 1 & 917 & 33,32 & - & - & - & - \\
\hline \multirow{2}{*}{$\begin{array}{l}\text { DUMMYGAAPETR X } \\
\text { DUMMYBTD }\end{array}$} & 0 & 2002 & 72,75 & - & - & - & - \\
\hline & 1 & 750 & 27,25 & - & - & - & - \\
\hline
\end{tabular}

Nota: INVESTIMENTO - Nível de investimento da firma; GAAPETR - Medida de agressividade fiscal; BTD - Book Tax Differences; TAM - Tamanho; $Q$ - Oportunidades de investimento da firma; ROA - Retorno operacional da empresa; FCO - Fluxo de Caixa Operacional; IDADE - Idade da firma; Z - Proxy para probabilidade de falência financeira da firma; TCR - Taxa de Crescimento da Receita da Firma; TAN - Tangibilidade dos ativos; END - Nível de Endividamento; DUMMYDIV - Assume o valor 1 se a firma paga dividendos, 0 caso contrário; DUMMYJSCP - Assume o valor 1 se a firma paga Juros Sobre o Capital Próprio, 0 caso contrário; DUMMYGAAPETR - Assume o valor 1 se a medida de agressividade fiscal foi maior que a divisão da mediana do setor, 0 caso contrário; DUMMYBTD - Assume 1 se o Book Tax Differences for maior que a divisão da mediana do setor - a mínima pela máxima - a mínima, 0 caso contrário.

Fonte: Resultados da pesquisa.

Como pode ser observado na Tabela 3 algumas variáveis apresentaram correlação estatisticamente significativa, sugerindo problemas de multicolinearidade. Todavia, conforme Gujarati (2006) e Fávero \& Belfiore (2017), não necessariamente a presença de alta correlação entre as variáveis gera, necessariamente, "estimadores ruins ou fracos" e ainda "que a presença de multicolinearidade não significa que o modelo possui problemas" (Fávero \& Belfiore, 2017, p. 555). Ademais, a Regressão Quantílica "não tem como pressuposto a existência de normalidade dos resíduos" (Fávero \& Belfiore, 2017, p. 603). 
TABELA 3: Correlação entre as variáveis dependente e preditoras (Continua)

\begin{tabular}{|c|c|c|c|c|c|c|c|}
\hline & INVESTIMENTO & GAAP ETR & BTD & TAM & Q & ROA & FCO \\
\hline INVESTIMENTO & 1,000 & & & & & & \\
\hline GAAP ETR & $0,114^{* *}$ & 1,000 & & & & & \\
\hline BTD & $-0,016$ & 0,012 & 1,000 & & & & \\
\hline TAM & $0,055^{* *}$ & $0,114^{* *}$ & $0,056^{* *}$ & 1,000 & & & \\
\hline Q & $0,047^{*}$ & $0,122^{* *}$ & $0,161^{* *}$ & $0,184^{* *}$ & 1,000 & & \\
\hline ROA & $0,198^{* *}$ & $0,351^{* *}$ & $0,360^{* *}$ & $0,142^{* *}$ & $0,410^{* *}$ & 1,000 & \\
\hline FCO & 0,033 & $0,162^{* *}$ & $0,214^{* *}$ & $0,067^{* *}$ & $0,303^{* *}$ & $0,495^{* *}$ & 1,000 \\
\hline IDADE & $-0,017$ & $-0,086^{* *}$ & $-0,024$ & $-0,171^{* *}$ & $-0,127^{* *}$ & $-0,109^{* *}$ & $-0,064^{* *}$ \\
\hline Z & $0,092^{* *}$ & $0,348^{* *}$ & $0,360^{* *}$ & $0,139^{* *}$ & $0,472^{* *}$ & $0,609^{* *}$ & $0,361^{* *}$ \\
\hline TCR & $0,210^{* *}$ & $0,197^{* *}$ & $0,078^{* *}$ & $0,073^{* *}$ & $0,171^{* *}$ & $0,354^{* *}$ & $0,153^{* *}$ \\
\hline TAN & $0,177^{* *}$ & 0,011 & $-0,152^{* *}$ & $-0,159^{* *}$ & $-0,013$ & $-0,024$ & 0,022 \\
\hline END & $-0,127^{* *}$ & $-0,294^{* *}$ & $-0,191^{* *}$ & $-0,035$ & $-0,007$ & $-0,236^{* *}$ & $-0,186^{* *}$ \\
\hline GAAP ETRX BTD & $-0,070^{* *}$ & $-0,323^{* *}$ & $0,132^{* *}$ & $-0,041^{*}$ & $-0,031$ & $-0,130^{* *}$ & $-0,034$ \\
\hline
\end{tabular}

INVESTIMENTO - Nível de investimento da firma; GAAPETR - Medida de agressividade fiscal; BTD - Book Tax Differences;

TAM - Tamanho; $Q$ - Oportunidades de investimento da firma; ROA - Retorno operacional da empresa; FCO - Fluxo de caixa operacional; IDADE - Idade da firma; Z - Proxy para probabilidade de falência financeira da firma; TCR - Taxa de Crescimento da Receita da Firma; TAN - Tangibilidade dos ativos; END - Nível de Endividamento

(*) Estatisticamente significante ao nível de 0.01; (**) Estatisticamente significante ao nível de 0.05; Correlação de Spearman

TABELA 3: Correlação entre as variáveis dependente e preditoras (Conclusão)

\begin{tabular}{|c|c|c|c|c|c|c|}
\hline & IDADE & $Z$ & TCR & TAN & END & GAAP ETR X BTD \\
\hline IDADE & 1,000 & & & & & \\
\hline$Z$ & $-0,083^{* *}$ & 1,000 & & & & \\
\hline TCR & $-0,101^{* *}$ & $0,252^{* *}$ & 1,000 & & & \\
\hline TAN & $0,144^{* *}$ & $-0,106^{* *}$ & $-0,001$ & 1,000 & & \\
\hline END & $0,094^{* *}$ & $-0,513^{* *}$ & $-0,070^{* *}$ & $0,053^{* *}$ & 1,000 & \\
\hline GAAP ETR X BTD & $-0,008$ & $-0,149^{* *}$ & $-0,076^{* *}$ & $0,040^{*}$ & $0,138^{* *}$ & 1,000 \\
\hline
\end{tabular}

INVESTIMENTO - Nível de investimento da firma; GAAPETR - Medida de agressividade fiscal; BTD - Book Tax Differences; TAM - Tamanho; Q - Oportunidades de investimento da firma; ROA - Retorno operacional da empresa; FCO - Fluxo de caixa operacional; IDADE - Idade da firma; Z - Proxy para probabilidade de falência financeira da firma; TCR - Taxa de Crescimento da Receita da Firma; TAN - Tangibilidade dos ativos; END - Nível de Endividamento

(*) Estatisticamente significante ao nível de 0.01; (**) Estatisticamente significante ao nível de 0.05; Correlação de Spearman

A Tabela 4 a seguir apresenta as significâncias estatísticas das variáveis por modelo com a finalidade de sintetizar os dados e facilitar as análises:

Tabela 4 - Significância dos modelos nos quantis $25\left(^{*}\right), 50\left(^{* *}\right)$ e $75(* * *)$

\begin{tabular}{|c|c|c|c|c|c|c|c|c|}
\hline Variáveis & 1 & 2 & 3 & 4 & 5 & 6 & 7 & 8 \\
\hline INVESTIMENTO & & & & & & & & \\
\hline GAAPETR & $*$ & $*$ & & & & & & \\
\hline BTD & & & & & & & & \\
\hline GAAPETR*BTD & & & & & & & & \\
\hline DUMmYBTD & & & & & & & & \\
\hline DUMMYGAAPETR & & & & & & $(-)^{* * *}$ & & \\
\hline DUMMYGAAPETR*DUMMYBTD & & & & & & & & $*$ \\
\hline TAM & $* * *$ & $* * *$ & $* * *$ & $* * *$ & $* * *$ & $* * *$ & $* * *$ & \\
\hline *** & & & & & & & & \\
\hline Q & $*$ & & & & & & & \\
\hline ROA & $* * *$ & $* * *$ & & $* * *$ & $* * *$ & $* * *$ & & $* * *$ \\
\hline FCO & & & & & & & & \\
\hline
\end{tabular}




\begin{tabular}{|c|c|c|c|c|c|c|c|c|}
\hline IDADE & & $*$ & $*$ & $* * * *$ & & & $*$ & \\
\hline Z & & & & & & $(-)^{* *}$ & & \\
\hline TCR & $*$ & $*$ & & $*$ & $(-)^{* * *}$ & $(-)^{* *}$ & $(-)^{* * *}$ & $*$ \\
\hline TAN & $* * *$ & & $* * *$ & $* * *$ & $* * *$ & $* * *$ & $* * *$ & $* * * * *$ \\
\hline END & $(-)^{* * * * *}$ & $(-)^{*}$ & $(-)^{* * *}$ & $(-)^{* * * * *}$ & $* * *$ & $(-)^{* * *}$ & $(-)^{* * * * *}$ & $(-)^{* * * * *}$ \\
\hline DUMMYDIV & $(-)^{*}$ & $(-)^{*}$ & & $(-)^{*}$ & $* * *$ & $* * *$ & & $(-)^{*}$ \\
\hline DUMMYJSCP & $(-)^{* * *}$ & & $(-)^{* * *}$ & $(-)^{* * *}$ & $(-)^{* * *}$ & $(-)^{* *}$ & & $(-)^{* * *}$ \\
\hline
\end{tabular}

Notas: (-): significância negativa. As aspas (") separam significâncias em mais de um quantil. Fonte: Resultados da pesquisa.

'Observe-se que a maioria das variáveis apresenta significância estatística com relação positiva com a variável investimento, ou seja, um aumento nessas variáveis corresponde a um aumento dos investimentos. Já as variáveis com o sinal de negativo (-) indicam que o aumento dessas variáveis corresponde a uma diminuição dos investimentos.

É importante destacar a significância estatística para a variável dependente GAAPETR e a interação das Dummies GAAPETR e BTD, o que demonstra que há relação entre a taxa de tributação efetiva e o acréscimo dos investimentos, corroborando a hipótese da presente pesquisa. Outro aspecto que merece destaque é a significância das variáveis de controle, pois indica a presença de custos não tributários.

A Tabela 5 a seguir permite a comparação dos resultados dos testes estatísticos com pesquisas anteriores que investigaram a agressividade fiscal e o nível e eficiência dos investimentos das empresas:

Tabela 5 - Correlação com outros modelos

\begin{tabular}{|c|c|c|c|c|c|c|c|}
\hline & \multicolumn{2}{|c|}{ Goldman (2016) } & \multirow{2}{*}{$\begin{array}{c}\text { Mayberry (2012) } \\
-\end{array}$} & \multirow{2}{*}{$\frac{\text { Biddle et al. (2009) }}{-}$} & \multirow{2}{*}{$\begin{array}{c}\text { Cheng et al. (2013) } \\
-\end{array}$} & \multirow{2}{*}{$\begin{array}{c}\text { Blouin, et al. (2012) } \\
-\end{array}$} & \multirow{2}{*}{$\begin{array}{l}\text { Alencastre et al. (2018) } \\
-\end{array}$} \\
\hline- & ETR & BTD & & & & & \\
\hline INVESTIMENTO & & & $* * *$ & NA & NA & NA & \\
\hline GAAPETR & & & $* * *$ & & & & \\
\hline \multicolumn{8}{|l|}{ BTD } \\
\hline TAM & *** & *** & $* *$ & *** & ** & *** & \\
\hline $\mathrm{Q}$ & *** & *** & & & *** & & \\
\hline ROA & & & & & & $* * *$ & *** \\
\hline $\mathrm{FCO}$ & & ** & $* * *$ & $* * *$ & ** & & \\
\hline IDADE & *** & *** & $* * *$ & *** & *** & & \\
\hline z & ** & ** & $* * *$ & $* * *$ & & & \\
\hline TCR & & ** & & *** & *** & *** & *** \\
\hline TAN & *** & **** & $* * *$ & *** & *** & & \\
\hline END & & & $* * *$ & $* * *$ & & *** & ** \\
\hline DUMMYDIV & $* *$ & $* * *$ & $* * *$ & $* * *$ & $* *$ & & \\
\hline DUMMYJSCP & $* *$ & $* * *$ & $* * *$ & $* * *$ & $* *$ & & \\
\hline
\end{tabular}

Fonte: Resultados da pesquisa.

Os modelos 3, 4, 5 e 7 corroboram, em sua maior parte, os achados de Goldman (2016) e Blaylock et al. (2016) que não encontraram significância estatística positiva para as variáveis de agressividade fiscais propostas na presente pesquisa e de Alencastre et al (2018) que observaram que o investimento não é afetado pelas métricas de agressividade fiscal por eles adotadas.

A variável de controle FCO não apresentou significância estatística em nenhum dos modelos, não contribuindo para a análise da relação entre agressividade fiscal e investimento. A variável $Z$ apresentou significância em apenas um modelo, o que sugere pouco poder de explicação sobre o investimento, assim como a variável Q. Nos modelos 2, 3, 4 e 7 a variável IDADE apresentou significância estatística. 
Este resultado é o mesmo observado por Goldman (2016), Mayberry (2012), Biddle et al. (2009) e Cheng et al. (2013), que tiveram significância da mesma variável a 1\% de significância, sugerindo que quanto mais consolidada for a empresa maior o seu impacto sobre o investimento.

TCR, TAN, END, DUMMYDIV e DUMMYJSCP apresentaram significância estatística em alguns modelos e correlação com pesquisas anteriores. Aqui, cabe o destaque que END, TCR e ROA tiveram significância nas pesquisas de Blouin et al. (2012) e Alencastre et al. (2018), o que demonstra que alavancagem, receita e retorno têm impactos positivos no investimento. Cabe o destaque para a variável de controle Tamanho (TAM), que apresentou significância estatística em todos os modelos, sendo que apenas a pesquisa de Alencastre et al. (2018) não constatou significância para esta variável da maturidade da companhia.

Com relação às variáveis dependentes, a presente pesquisa corrobora os resultados de Mayberry (2012) ao obter significância para GAAPETR nos modelos 1 e 2, assim como para DUMMYGAAPETR e a interação das dummies das variáveis dependentes. Enquanto que para as variáveis de controle obteve-se correlação próxima a pesquisas anteriores em maior ou menor grau, de acordo com a significância estatística.

\section{CONSIDERAÇÕES FINAIS}

O objetivo do estudo foi analisar a relação das práticas de agressividade fiscal com o investimento das empresas. Analisou-se dados de 4.764 empresas de 13 setores econômicos no período de 2010 a 2018, totalizando 2.752 observações, por meio de Regressão Quantílica.

Os resultados permitiram concluir, em particular para as variáveis GAAPETR, nos modelos 1 e 2, interação entre DUMMYGAAPETR e DUMMYBTD, no modelo 8, e DUMMYGAAPETR, no modelo 6, que a agressividade fiscal tem impacto positivo no investimento das empresas, o que ratifica a hipótese de pesquisa e corrobora os achados de Mayberry (2012).

Reforçando os achados desta pesquisa o planejamento tributário agressivo está relacionado ao investimento das empresas devido a duas imperfeições do mercado de capitais: seleção adversa e risco moral (Jensen, 1986; Jensen \& Meckling, 1976; Stein, 2003). Portanto, o paradigma SW de que a agressividade fiscal atua como um custo não tributário significativo do investimento é aceitável, uma vez que, devido ao aumento das informações assimétricas produzidas por essas atividades, há relativa diminuição da transparência contábil (Desai \& Dharmapala, 2007).

Considerando empresas mais eficientes dentro de uma estratégia de planejamento tributário agressivo, os custos de seus impostos tendem a ter menos relevância, conforme Hanlon e Slemrod (2009). Empresas com taxas de GAAPETR maiores têm práticas tributárias menos agressivas (Martinez, 2017, p. 114 ) e, portanto, restam menos recursos financeiros para serem canalizadas para o investimento. Como houve significância, é válido afirmar que a agressividade fiscal atua como um custo não tributário, com base no paradigma SW de todos os custos, com influência no investimento da empresa, atuando como um custo de agência.

A presente pesquisa apresenta limitações em relação ao tamanho da amostra analisada e em relação às proxies de agressividade fiscal, ainda em estágio de desenvolvimento e aperfeiçoamento, embora esta seja uma dificuldade recorrente nos trabalhos sobre o tema, como bem observaram Chiachio \& Martinez (2019).

Para a investigação de aspectos adicionais, sugere-se aprimorar a estrutura analítica, a fim de incorporar a interação com decisões de períodos anteriores; o teste da relação com a utilização de outras proxies 
de agressividade tributária, como forma de se obter um resultado mais robusto; investigar a interação entre a agressividade fiscal e variáveis de qualidade contábil; avaliar os efeitos dos planos de remuneração variável dos gerentes sobre a agressividade tributária, bem como a interação entre bonificação, evasão tributária e probabilidade de detecção.

\section{REFERÊNCIAS}

Alencastre, B. Z., da Silva Campos, B., \& Marques, V. A. (2018). O impacto da Agressividade Tributária Sobre o Nível de Investimentos, Eficiência Produtiva e Rentabilidade de Empresas Listadas na $[B]^{3}$.

Allingham, M. G., \& Sandmo, A. (1972). Income tax evasion: A theoretical analysis. Journal of public economics, 1(3-4), 323-338. Disponível em: https://doi.org/10.1016/0047-2727(72)90010-2. Acesso em: ago. 2020.

Almeida, H., \& Campello, M. (2007). Financial Constraints, Asset Tangibility, and Corporate Investment. Review of Financial Studies, 20(5), 1429-1460. Disponível em: https://doi.org/10.1093/rfs/hhm019. Acesso em: ago. 2020.

Altman, E. I. (1968). Financial ratios, discriminant analysis and the prediction of corporate bankruptcy. The journal of finance, 23(4), 589-609. DOI 10.2307/2978933. Disponível em: https://www.jstor.org/ stable/2978933. Acesso em: ago. 2020.

Araújo, R. A. de M., Santos, L. M. da S., Leite Filho, P. A. M., \& Camara, R. P. de B. (2018). Agressividade Fiscal: Uma comparação entre empresas listadas na NYSE e BM\&FBOVESPA1. Enfoque: Reflexão Contábil, 37(1), 39-54. Disponível em: https://doi.org/10.4025/enfoque.v37i1.32926. Acesso em: ago. 2020.

Balakrishnan, K., Core, J. E., \& Verdi, R. S. (2014). The relation between reporting quality and financing and investment: Evidence from changes in financing capacity. Journal of Accounting Research, 52(1), 1-36. Disponível em: https://doi.org/10.1111/1475-679X.12031. Acesso em: ago. 2020.

Biddle, G. C., \& Hilary, G. (2006). Accounting Quality and Firm-Level Capital Investment. The Accounting Review, 81(5), 963-982. Disponível em: https://doi.org/10.2308/accr.2006.81.5.963. Acesso em: ago. 2020.

Biddle, G. C., Hilary, G., \& Verdi, R. S. (2009). How does financial reporting quality relate to investment efficiency? Journal of Accounting and Economics, 48(2-3), 112-131. Disponível em: https://doi.org/10.1016/j. jacceco.2009.09.001. Acesso em: ago. 2020.

Blaylock, B. S. (2016). Is Tax Avoidance Associated with Economically Significant Rent Extraction among U.S. Firms? Contemporary Accounting Research, 33(3), 1013-1043. Disponível em: https://doi. org/10.1111/1911-3846.12174. Acesso em: ago. 2020.

Blouin, J. L., Devereux, M., \& Shackelford, D. A. (2012). Investment, tax uncertainty, and aggressive tax avoidance. Documento de trabajo, Oxford University. 
Caldeira, J., \& Loncan, T. (2013). Capital Structure, Cash Holdings and Firm Value: A Study of Brazilian Listed Firms. SSRN Electronic Journal. Disponível em: https://doi.org/10.2139/ssrn.2329346. Acesso em: ago. 2020.

Cheng, M., Dhaliwal, D., \& Zhang, Y. (2013). Does investment efficiency improve after the disclosure of material weaknesses in internal control over financial reporting? Journal of Accounting and Economics, 56(1), 1-18. Disponível em: https://doi.org/10.1016/j.jacceco.2013.03.001. Acesso em: ago. 2020.

Chiachio, V. F. de O., \& Martinez, A. L. (2019). Efeitos do Modelo de Fleuriet e Índices de Liquidez na Agressividade Tributária. Revista de Administração Contemporânea, 23(2), 160-181. https://doi.org/10.1590/1982-7849rac2019180234

Crabtree, A. D., \& Kubick, T. R. (2014). Corporate tax avoidance and the timeliness of annual earnings announcements. Review of Quantitative Finance and Accounting, 42(1), 51-67. Disponível em: https:// doi.org/10.1007/s11156-012-0333-9. Acesso em: ago. 2020.

Desai, M. A., \& Dharmapala, D. (2006). Corporate tax avoidance and high-powered incentives. Journal of Financial Economics, 79(1), 145-179. Disponível em: https://doi.org/10.1016/j.jfineco.2005.02.002. Acesso em: ago. 2020.

Desai, M. A., \& Dharmapala, D. (2007). Taxation and Corporate Governance: An Economic Approach. SSRN Electronic Journal. Disponível em: https://doi.org/10.2139/ssrn.983563. Acesso em: ago. 2020.

Fávero, L. P. \& Belfiore, P. (2017). Manual de Análise de Dados. Rio de Janeiro: Elsevier.

Frank, M. M., Lynch, L. J., \& Rego, S. O. (2009). Tax Reporting Aggressiveness and Its Relation to Aggressive Financial Reporting. The Accounting Review, 84(2), 467-496. Disponível em: https://doi.org/10.2308/ accr.2009.84.2.467. Acesso em: ago. 2020.

Goh, B. W., Lee, J., Lim, C. Y., \& Shevlin, T. (2016). The Effect of Corporate Tax Avoidance on the Cost of Equity. The Accounting Review, 91(6), 1647-1670. Disponível em: https://doi.org/10.2308/accr-51432. Acesso em: ago. 2020.

Goldman, N. C. (2016). The effect of tax aggressiveness on investment efficiency. Disponível em: https:// repository.arizona.edu/handle/10150/612100. Acesso em: ago. 2020.

Graham, J. R., Hanlon, M., Shevlin, T., \& Shroff, N. (2014). Incentives for Tax Planning and Avoidance: Evidence from the Field. The Accounting Review, 89(3), 991-1023. Disponível em: https://doi.org/10.2308/ accr-50678. Acesso em: ago. 2020.

Gujarati, D. (2006). Econometria Básica, 4 ed. Trad. Maria José Cyhlar Monteiro. Rio de Janeiro: Elsevier.

Hanlon, M., \& Slemrod, J. (2009). What does tax aggressiveness signal? Evidence from stock price reactions to news about tax shelter involvement. Journal of Public Economics, 93(1-2), 126-141. Disponível em: https://doi.org/10.1016/j.jpubeco.2008.09.004. Acesso em: ago. 2020. 
Jensen, M. C., \& Meckling, W. H. (1994). The Nature of Man. Journal of Applied Corporate Finance, 7(2), 4-19. Disponível em: https://doi.org/10.1111/j.1745-6622.1994.tb00401.x. Acesso em: ago. 2020.

Jensen, M. C., \& Meckling, W. H. (1976). Theory of the firm: Managerial behavior, agency costs and ownership structure. Journal of financial economics, 3(4), 305-360. Disponível em: https://doi.org/10.1016/ 0304-405X(76)90026-X. Acesso em: ago. 2020.

Jensen, M. C. (1986). Agency Costs of Free Cash Flow, Corporate Finance, and Takeovers. The American Economic Review, 76(2), 323-329. Disponível em: https://doi.org/10.2139/ssrn.99580. Acesso em: ago. 2020.

Kleinbard, E. D. (2013). Through a latte, darkly: Starbucks's stateless income planning. Tax Notes, 15151535. Disponível em: https://ssrn.com/abstract=2264384. Acesso em: ago. 2020.

Koenker, R., \& Bassett, G. (1978). Regression Quantiles. Econometrica, 46(1), 33. Disponível em: https:// doi.org/10.2307/1913643. Acesso em: ago. 2020.

Lietz, G. M. (2013). Tax Avoidance vs. Tax Aggressiveness: A Unifying Conceptual Framework. SSRN Electronic Journal. Disponível em: https://doi.org/10.2139/ssrn.2363828. Acesso em: ago. 2020.

Lisowsky, P., Robinson, L., \& Schmidt, A. (2010). An examination of FIN 48: Tax shelters, auditor independence, and corporate governance. University of Illinois at Urbana-Champaign working paper.

Martinez, A. L., \& Paste Junior, E. (2018). The Relation Between Operational Efficiency and Tax Aggressiveness in Brazil. Disponível em: https://doi.org/10.2139/ssrn.3327787. Acesso em: ago. 2020.

Martinez, A. L., \& Ramalho, G. C. (2014). Family Firms and Tax Aggressiveness in Brazil. International Business Research, 7(3). Disponível em: https://doi.org/10.5539/ibr.v7n3p129. Acesso em: ago. 2020.

Martinez, A. L., \& Silva, R. F. (2017). Agressividade Fiscal e o Custo de Capital de Terceiros no Brasil. Revista de Gestão, Finanças e Contabilidade, 7(1), 240-251. Disponível em: https://doi.org/10.18028/2238-5320/ rgfc.v7n1p240-251. Acesso em: ago. 2020.

Martinez, A. L. (2017). Agressividade Tributária: Um Survey da Literatura. Revista de Educação e Pesquisa em Contabilidade (REPeC), 11(0), 106-124. Disponível em: https://doi.org/10.17524/repec.v11i0.1724. Acesso em: ago. 2020.

Mayberry, M. (2012). Tax avoidance and investment: Distinguishing the effects of capital rationing and overinvestment. Tese de Doutorado.

Park, J., Ko, C. Y., Jung, H., \& Lee, Y.-S. (2016). Managerial ability and tax avoidance: Evidence from Korea. Asia-Pacific Journal of Accounting \& Economics, 23(4), 449-477. Disponível em: https://doi.org/10.1080 /16081625.2015.1017590. Acesso em: ago. 2020. 
Robinson, J. R., Sikes, S. A., \&Weaver, C. D. (2010). Performance Measurement of Corporate Tax Departments. The Accounting Review, 85(3), 1035-1064. Disponível em: https://doi.org/10.2308/accr.2010.85.3.1035. Acesso em: ago. 2020.

Sachsida, A., Mendonca, M. J. C., \& Moreira, T. B. S. (2016). Tax and growth in a developing country: The case of Brazil. Disponível em: https://doi.org/10.2139/ssrn.2772161. Acesso em: ago. 2020.

Shackelford, D. A., \& Shevlin, T. (2001). Empirical tax research in accounting. Journal of accounting and economics, 31(1-3), 321-387. Disponível em: https://doi.org/10.1016/S0165-4101(01)00022-2. Acesso em: ago. 2020.

Scholes, M. S., Wilson, G. P., \& Wolfson, M. A. (1990). Tax Planning, Regulatory Capital Planning, and Financial Reporting Strategy for Commercial Banks. The Review of Financial Studies, 3(4), 625-650. Disponível em: https://doi.org/10.1093/rfs/3.4.625. Acesso em: ago. 2020.

Scholes, M. S., Wolfson, M. A., Erickson, M. M., Hanlon, M. L., Maydew, E. L., \& Shevlin, T. (2015). Taxes and business strategy: A planning approach (Fifth edition). Pearson.

Slemrod, J. (2004). The economics of corporate tax selfishness. National Bureau of Economic Research. DOI 10.3386/w10858. Disponível em: https://doi.org/10.3386/w10858. Acesso em: ago. 2020.

Stein, J. C. (2003). Chapter 2-Agency, Information and Corporate Investment. In G. M. Constantinides, M. Harris, \& R. M. Stulz (Orgs.), Handbook of the Economics of Finance (Vol. 1, p. 111-165). Elsevier. Disponível em: https://doi.org/10.1016/S1574-0102(03)01006-9. Acesso em: ago. 2020.

Theóphilo, C. R., \& Martins, G. de A. (2009). Metodologia da investigação científica para ciências sociais aplicadas. São Paulo: Atlas, 2(104-119), 25.

Tobin, J. (1969). A General Equilibrium Approach To Monetary Theory. Journal of Money, Credit and Banking, 1(1), 15. Disponível em: https://doi.org/10.2307/1991374. Acesso em: ago. 2020.

Zimmerman, J. L. (1983). Taxes and firm size. Journal of Accounting and Economics, 5, 119-149. Disponível em: https://doi.org/10.1016/0165-4101(83)90008-3. Acesso em: ago. 2020. 